\title{
ctDNA facilitated the diagnosis of a patient with synchronous urothelial carcinoma and non-small cell lung cancer: case report
}

\author{
Chengyuan Qian ${ }^{1}$, Nan Dai ${ }^{1}$, Mingfang Xu' ${ }^{1}$, Hao Luo ${ }^{1}$, Yan Feng ${ }^{1}$, Min Zhang ${ }^{2}$, Rongrong Chen ${ }^{2}$, \\ Dong Wang ${ }^{1}$
}

${ }^{1}$ Department of Cancer Center, Daping Hospital \& Army Medical Center of the PLA, Chongqing, China; ${ }^{2}$ Geneplus-Beijing, Beijing, China Correspondence to: Dong Wang. Department of Cancer Center, Daping Hospital \& Army Medical Center of PLA, No. 10 Changjiang Zhi Road, Yuzhong District, Chongqing, China. Email: dongwang64@hotmail.com.

\begin{abstract}
The diagnosis and treatment for multiple primary cancers have been a great challenge in clinical practice. Circulating tumor DNA (ctDNA) is tumor-derived fragmented DNA that circulates in the blood. Herein we report a case that ctDNA facilitated the diagnosis of synchronous urothelial carcinoma (UC) and lung adenocarcinoma. A 58-year-old male patient was diagnosed with UC initially. Computed tomography (CT) revealed multiple metastases without the brain after surgery and adjuvant chemotherapy. However, the patient had a progressively worsened headache symbol during system therapy. We explored the genome variations using next-generation sequencing (NGS). HRAS and TP53 mutations were detected from UC surgical tissue and postoperative ctDNA. Unexpectedly, the epidermal growth factor receptor (EGFR) exon 19 deletion (19del) mutation, which is common in non-small cell lung cancer (NSCLC), was also identified in ctDNA. Pathological analysis of a neck lymph node confirmed adenocarcinoma derived from the lung. Meanwhile, EGFR 19del was detected in neck lymph node biopsy. The ctDNA contained both UC and lung adenocarcinoma-derived mutations. Thus, the diagnosis was modified into synchronous UC and lung adenocarcinoma. Interestingly, the lung adenocarcinoma-derived lesions responded well to osimertinib $(80 \mathrm{mg}$, once daily), while the UC did not. His headache rapidly subsided and disappeared. This case demonstrates that ctDNA analysis may better capture the molecular heterogeneity harbored by multiple primary tumors in a patient and can facilitate the diagnosis and therapy of patients with simultaneous cancers.
\end{abstract}

Keywords: Circulating tumor DNA (ctDNA); simultaneous cancer; non-small cell lung cancer (NSCLC); urothelial carcinoma (UC); epidermal growth factor receptor (EGFR); case report

Submitted Sep 22, 2020. Accepted for publication Oct 11, 2020.

doi: $10.21037 /$ atm-20-6552

View this article at: http://dx.doi.org/10.21037/atm-20-6552

\section{Background}

Lung cancer and urothelial carcinoma (UC) are major types of male cancer (1). Cancer of renal pelvis is relatively rare in renal tumors and UC. Treatment for advanced UC focus on cisplatin-based chemotherapy and anti-programmed cell death 1 (PD-1)/programmed cell death ligand 1 (PDL1) inhibitors (2). Target therapy is suitable for patients who have an appropriate marker, such as fibroblast growth factor receptor (FGFR) alterations (3). Non-small cell lung cancer (NSCLC) accounts for $85 \%$ of lung cancer cases, and has a greater chance of being treated by target therapy. Epidermal growth factor receptor $(E G F R)$ is the most common targetable driver gene in NSCLC. Patients who have an $E G F R$-sensitive mutation can benefit from EGFR tyrosine kinase inhibitors (EGFR-TKIs) but not PD-1/ PD-L1 inhibitors (4). Anti-PD-1/PD-L1 monotherapy or combination therapy is mainly used for patients whose driver gene negative and PD-L1 positive (5).

Epidemiological studies indicate that the prevalence of multiple primary cancer is approximately $2-17 \%$, with an increasing trend in recent years (6). The diagnosis and treatment for these patients have been a considerable challenge. Combination with urine cytology and cystoscopy is widely used to assess for the presence of UC. The 


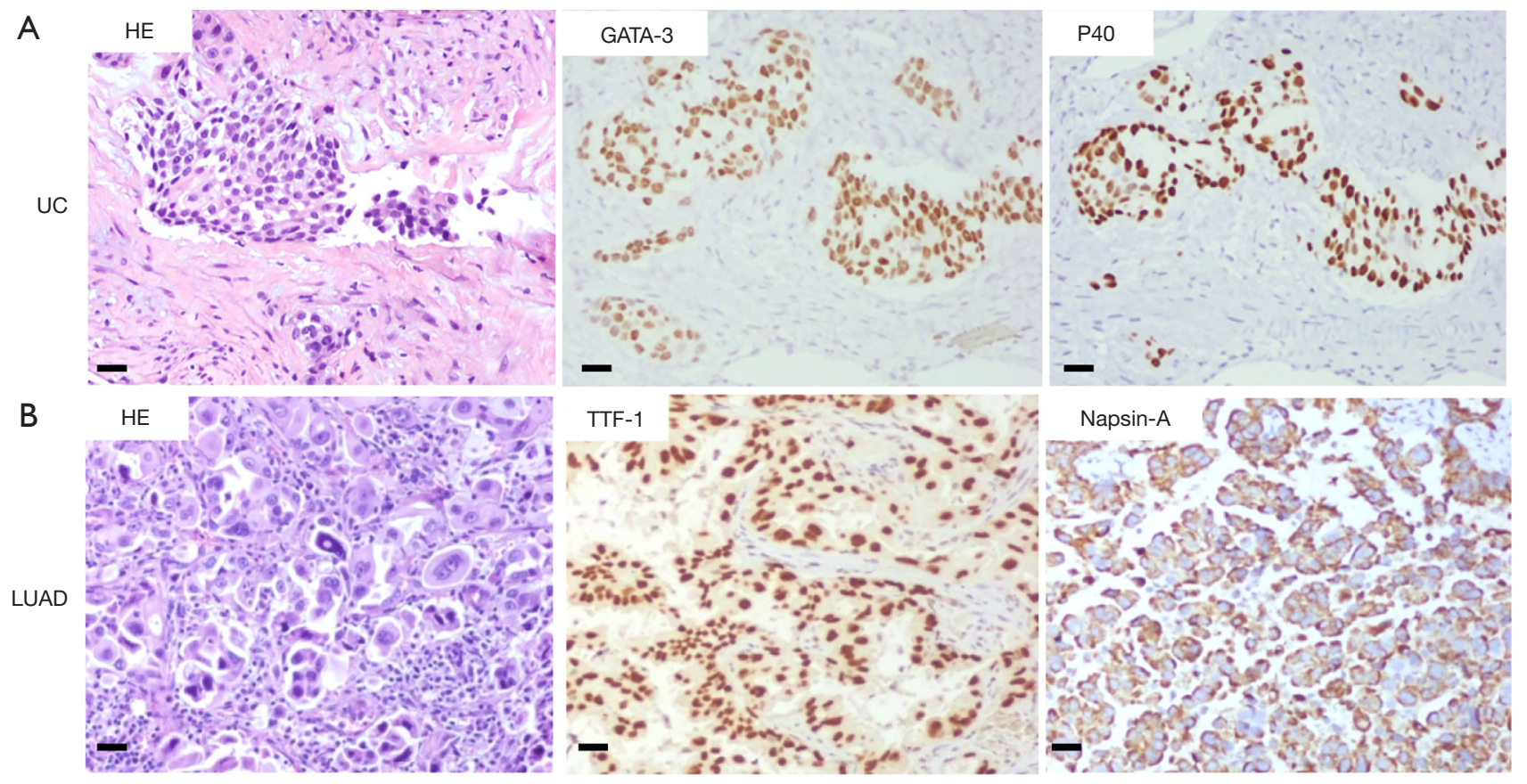

Figure 1 Hematoxylin and eosin (HE) and immunohistochemistry (IHC) staining of UC and NSCLC $(200 \times$, scale bar $=50 \mu m)$. (A) High grade urothelial carcinoma: GATA binding protein 3 (GATA-3) and Delta Np63 (P40); (B) lung adenocarcinoma (LUAD): thyroid transcription factor 1 (TTF-1) and Napsin-A.

diagnosis of NSCLC is based on the pathologic evaluation of specimens from biopsy or surgical. In cancer patients, circulating tumor DNA (ctDNA) are released from cancer cells and had short half-life of an hour or less (7). These characteristics determine the potential applications of ctDNA in cancer management. CtDNA analyzing by next-generation sequencing (NGS) can be used not only to monitor disease relapse but also to determine the origin of primary lesions (8). CtDNA is a promising circulating biomarker in monitoring and individualized treatment of $\mathrm{UC}$ according to previous report (9). However, its use in clinical practice of simultaneous UC and NSCLC is uncommon.

Here, we report a case that ctDNA sequencing facilitated the diagnosis and treatment of simultaneous UC and NSCLC in a 58-year-old male patient which occurred in our department. We present the following case in accordance with the CARE reporting checklist (available at http://dx.doi.org/10.21037/atm-20-6552).

\section{Case presentation}

This study was approved by the institutional ethics review board of the Army Medical Center of the PLA.
All procedures performed in this study involving human participants were in accordance with the Declaration of Helsinki (as revised in 2013). The patient provided written informed consent for specimen collection, genetic testing, and use of this information for research purposes.

A 58-year-old male patient with 30-year smoking history came to our hospital complaining of headache in August 2018. The patient underwent right laparoscopic radical nephrectomy and gemcitabine adjuvant chemotherapy for high-grade UC of the renal pelvis in July 2018 (Figure 1A). Contrast-enhanced chest-abdomen-pelvis computed tomography (CT) revealed that liver, bone, lung, and lymph node metastasis. Magnetic resonance imaging (MRI) results were normal (Figure S1). Subsequently, two cycles of nabpaclitaxel and one cycle of nivolumab were administered. However, his headache progressively worsened while the MRI was still normal. Meanwhile, we performed genetic analysis of ctDNA and renal surgical lesions using NGS. There were six mutations in the tissue, including HRAS p.G12D and TP53 p.E224K, and these mutations were also found in ctDNA. Unexpectedly, another eight mutations were identified in ctDNA, including EGFR exon 19 deletion (19del) and amplification (Table 1). EGFR 19del 
Table 1 Tissue and ctDNA NGS results

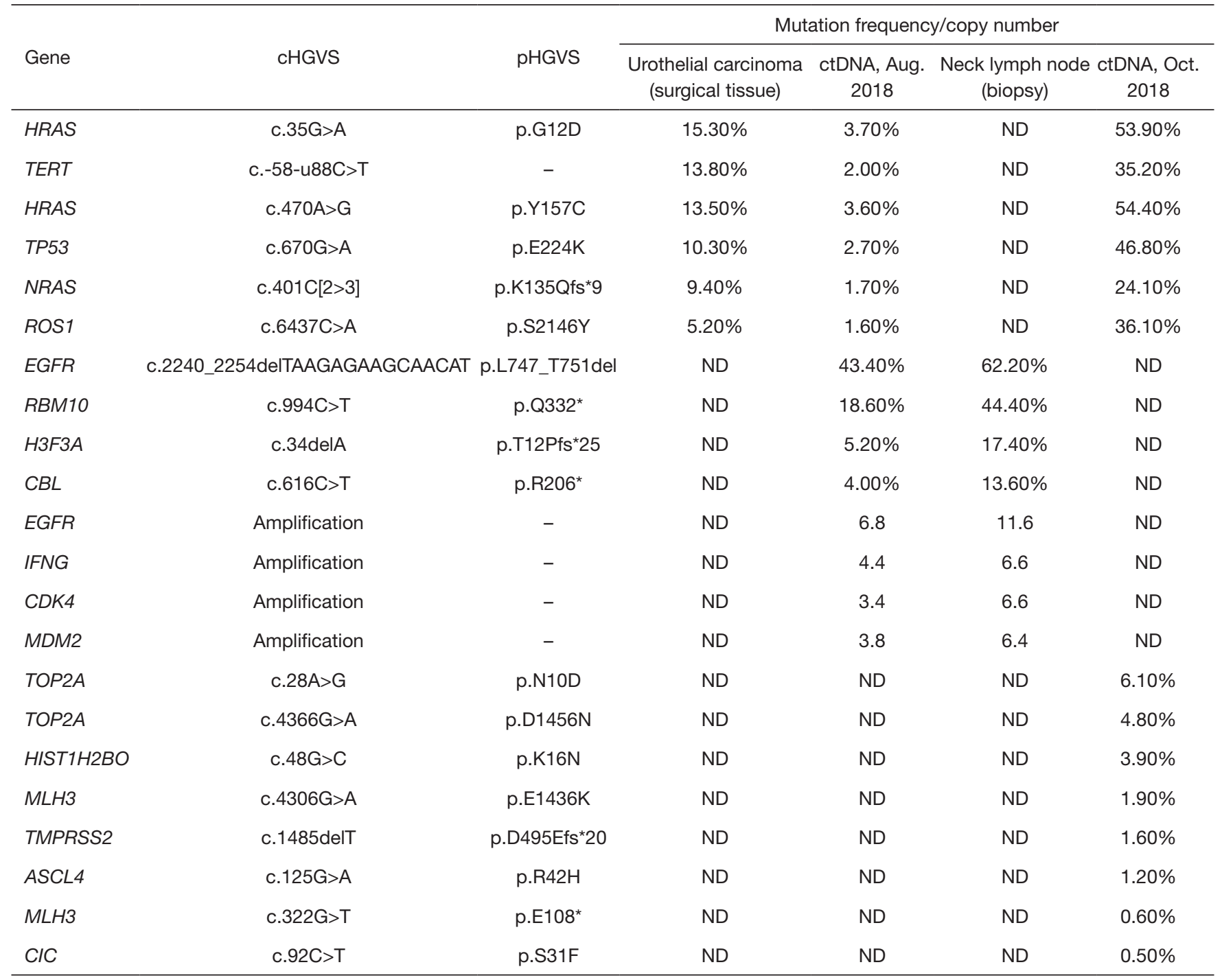

ctDNA, circulating tumor DNA; NGS, next-generation sequencing; ND, not detected.

mutation generally occurs in NSCLC and is sensitive to EGFR-TKIs (10). Suspecting a primary NSCLC, we performed a biopsy of the neck lymph node. The pathological characteristics suggested adenocarcinoma derived from the lung (Figure 1B). NGS for biopsies revealed eight mutations including EGFR $19 \mathrm{del}$ and amplification. Diagnosis changed to synchronous UC and lung adenocarcinoma, with multiple metastases. The patient subsequently received osimertinib (80 $\mathrm{mg}$, once daily) in September 2018. His headache rapidly subsided after 3 days and disappeared after 7 days. Carcinoembryonic antigen (CEA) levels continued to decreased. Contrast-enhanced chest-abdomen-pelvis CT results in October 2018 revealed that lung lesions and mediastinal lymph nodes had reduced, with all pericardial effusion being clearly absorbed. Liver, right abdomen, pelvic, and retroperitoneal lymph node metastasis and the number of bone metastases had increased (Figure 2), but mutations from lung adenocarcinoma had disappeared in ctDNA; however, the mutation from UC was still present and had increased significantly in frequency (Table 1). Unfortunately, the patient died 2 months later from massive bleeding in the digestive tract due to extremely low platelets and pancytopenia caused by extensive bone metastasis. 

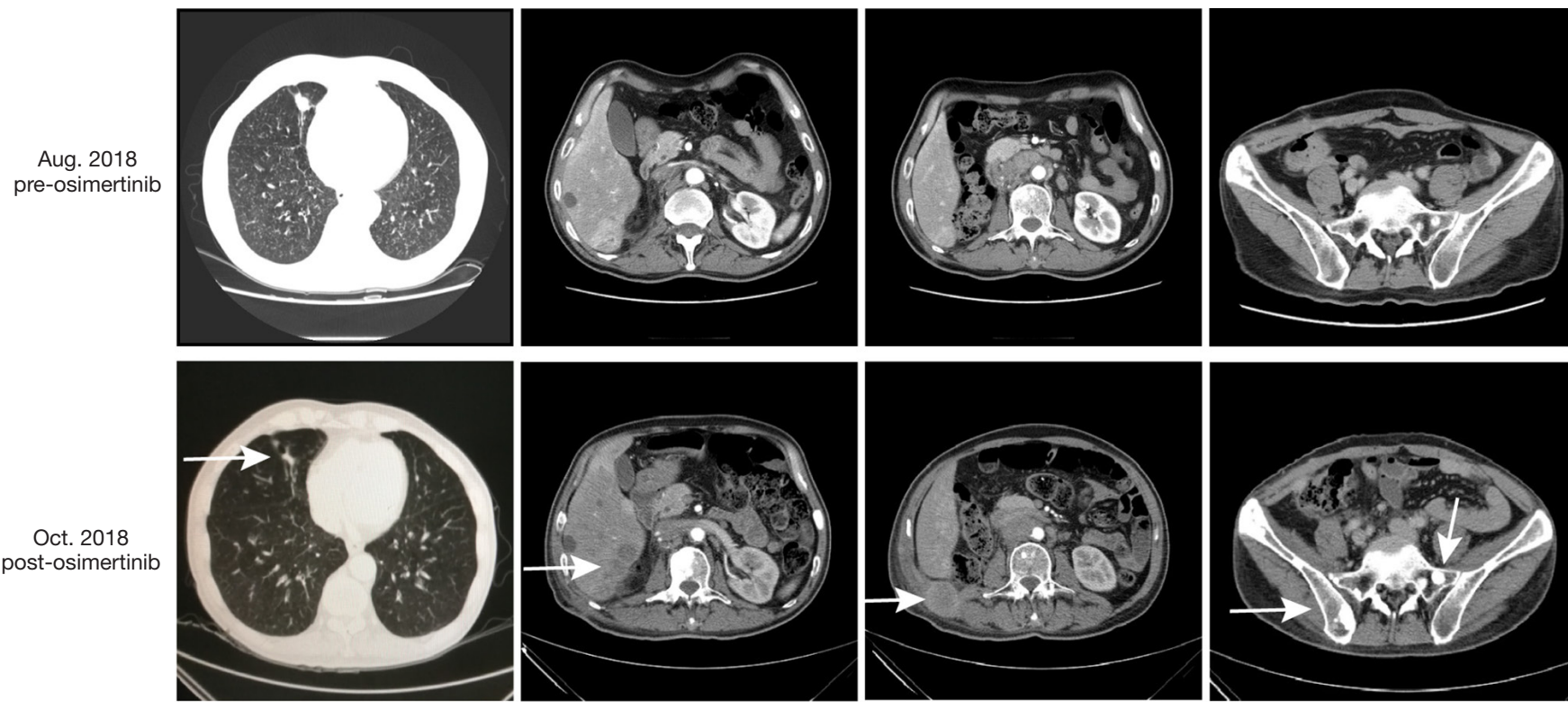

Figure 2 Contrast-enhanced chest-abdomen-pelvis computed tomography (CT) images before and after osimertinib. After osimertinib therapy, lung lesions and mediastinal lymph nodes had reduced; however, liver, right abdomen, pelvic, and retroperitoneal lymph node metastasis and the number of bone metastases had increased (white arrow).

\section{Discussion}

CtDNA has become a valuable source of sampling in precise cancer therapy, including molecular testing, and can provide new insights into intratumor and intertumor heterogeneity, thus aiding in cancer detection and monitoring. CtDNA can reflect the mutational landscape of tissue with favorable specificity and sensitivity, with increasing studies reporting high genomic concordance rates between tissue and plasma samples in NSCLC, UC, and other cancers (11-13). In early stage colorectal cancer, it was found that ctDNA can be used to detect residual tumor DNA following surgery and predict recurrence risk, with ctDNA-positive patients having higher recurrence rates and shorter disease-free survival than ctDNA-negative patients (14). For metastatic disease, ctDNA can be used to assess tumor burden, guide target therapy, and monitor disease response and resistance mechanisms, which includes EGFR-TKI management in EGFR-driven NSCLC (15).

In the current study, the patient was initially diagnosed as UC. HRAS and TP53 mutation were tested in surgical tissue and post-surgical plasma. TP53 and RAS-family gene are commonly mutated genes in UC, and ctDNApositive may predict poor survival. Unexpectedly, we also found EGFR 19del, EGFR amplification, and MDM2 amplification in ctDNA; as these usually occur NSCLC, we were alerted to the possibility of a double primary tumor. Pathological examination of the neck lymph node confirmed our suspicions, and the diagnosis was changed to synchronous UC and NSCLC. EGFR 19del being sensitive to EGFR-TKIs and MDM2 might have contributed to immunotherapy hyper-progression (16). CT imaging and ctDNA monitoring indicated osimertinib had a favorable effect on NSCLC disease; however, UC lesions continued to grow beyond control. Large-scale epidemiological studies have established an association between UC and NSCLC, indicating that the relative risk of developing NSCLC in UC patients increased (17-19). CtDNA analysis may be a feasible method for the detection of UC with lung metastasis or synchronous UC and NSCLC.

\section{Conclusions}

This case demonstrates that ctDNA analysis may better capture the molecular heterogeneity harbored by multiple primary tumors in a patient, and may thus provide useful information for detection, diagnosis, and therapy in patients who have simultaneous UC and NSCLC. Further studies still need to be developed to explore treatment strategies for 
simultaneous cancer.

\section{Acknowledgments}

We would like to express our deep gratitude to the patient and his family for permission to use the patient's information.

Funding: This research was funded by the National Natural Science Foundation of China (no. 81502241).

\section{Footnote}

Reporting Checklist: The authors have completed the CARE reporting checklist. Available at http://dx.doi.org/10.21037/ atm-20-6552

Conflicts of Interest: All authors have completed the ICMJE uniform disclosure form (available at http://dx.doi. org/10.21037/atm-20-6552). The authors have no conflicts of interest to declare.

Etbical Statement: The authors are accountable for all aspects of the work in ensuring that questions related to the accuracy or integrity of any part of the work are appropriately investigated and resolved. This study was approved by the institutional ethics review board of the Army Medical Center of the PLA. All procedures performed in this study involving human participants were in accordance with the Declaration of Helsinki (as revised in 2013). The patient provided written informed consent for specimen collection, genetic testing, and use of this information for research purposes.

Open Access Statement: This is an Open Access article distributed in accordance with the Creative Commons Attribution-NonCommercial-NoDerivs 4.0 International License (CC BY-NC-ND 4.0), which permits the noncommercial replication and distribution of the article with the strict proviso that no changes or edits are made and the original work is properly cited (including links to both the formal publication through the relevant DOI and the license). See: https://creativecommons.org/licenses/by-nc-nd/4.0/.

\section{References}

1. Siegel RL, Miller KD, Jemal A. Cancer statistics, 2019. CA Cancer J Clin 2019;69:7-34.

2. Koshkin VS, Basu A, Grivas P. The role of avelumab in advanced urothelial carcinoma in the context of a dynamic treatment landscape. Transl Cancer Res 2019;8:S130-2.

3. Loriot $Y$, Necchi A, Park SH, et al. Erdafitinib in Locally Advanced or Metastatic Urothelial Carcinoma. N Engl J Med 2019;381:338-48.

4. Rizvi H, Sanchez-Vega F, La K, et al. Molecular Determinants of Response to Anti-Programmed Cell Death (PD)-1 and Anti-Programmed Death-Ligand 1 (PD-L1) Blockade in Patients With Non-Small-Cell Lung Cancer Profiled With Targeted Next-Generation Sequencing. J Clin Oncol 2018;36:633-41.

5. Smit EF, de Langen AJ. Pembrolizumab for all PD-L1positive NSCLC. Lancet 2019;393:1776-8.

6. Vogt A, Schmid S, Heinimann K, et al. Multiple primary tumours: challenges and approaches, a review. ESMO Open 2017;2:e000172.

7. Corcoran RB, Chabner BA. Application of cell-free DNA analysis to cancer treatment. N Engl J Med 2018;379:1754-65.

8. Zhang S, Hu C, Fan J, et al. Detection and differential diagnosis of cancer relapse using circulating tumor DNA profiling in a patient with synchronous breast and rectal cancer. Cancer Biol Ther 2019;20:141-4.

9. Rink M, Schwarzenbach H, Riethdorf S, et al. The current role and future directions of circulating tumor cells and circulating tumor DNA in urothelial carcinoma of the bladder. World J Urol 2019;37:1785-99.

10. Bailey MH, Tokheim C, Porta-Pardo E, et al. Comprehensive characterization of cancer driver genes and mutations. Cell 2018;173:371-85.e18.

11. Reck M, Hagiwara K, Han B, et al. ctDNA determination of EGFR mutation status in European and Japanese patients with advanced NSCLC: the ASSESS study. J Thorac Oncol 2016;11:1682-9.

12. Kim SB, Dent R, Wongchenko MJ, et al. Concordance between plasma-based and tissue-based next-generation sequencing in LOTUS. Lancet Oncol 2017;18:e638.

13. Sundahl N, Vandekerkhove G, Decaestecker K, et al. Randomized phase 1 trial of pembrolizumab with sequential versus concomitant stereotactic body radiotherapy in metastatic urothelial carcinoma. Eur Urol 2019;75:707-11.

14. Reinert T, Henriksen TV, Christensen E, et al. Analysis of Plasma Cell-Free DNA by Ultradeep Sequencing in Patients With Stages I to III Colorectal Cancer. JAMA Oncol 2019;5:1124-31.

15. Mok T, Wu YL, Lee JS, et al. Detection and dynamic changes of EGFR mutations from circulating tumor 
DNA as a predictor of survival outcomes in NSCLC patients treated with first-line intercalated erlotinib and chemotherapy. Clin Cancer Res 2015;21:3196-203.

16. Kato S, Goodman A, Walavalkar V, et al. Hyperprogressors after immunotherapy: analysis of genomic alterations associated with accelerated growth rate. Clin Cancer Res 2017;23:4242-50.

17. Shiels MS, Gibson T, Sampson J, et al. Cigarette smoking prior to first cancer and risk of second smoking-associated cancers among survivors of bladder, kidney, head and neck, and stage I lung cancers. J Clin Oncol 2014;32:3989-95.

Cite this article as: Qian C, Dai N, Xu M, Luo H, Feng Y, Zhang M, Chen R, Wang D. ctDNA facilitated the diagnosis of a patient with synchronous urothelial carcinoma and non-small cell lung cancer. Ann Transl Med 2020;8(20):1323. doi: 10.21037/ atm-20-6552
18. Anderson JK, Alanee S, Lindgren B, et al., editors. The risk of bladder cancer in patients diagnosed with other primary neoplasms: analysis of the SEER database. Urologic Oncology: Seminars and Original Investigations, Elsevier; 2013.

19. Ferreccio C, Yuan Y, Calle J, et al. Arsenic, tobacco smoke, and occupation: associations of multiple agents with lung and bladder cancer. Epidemiology 2013;24:898-905 .

(English Language Editor: J. Gray) 


\section{Supplementary}

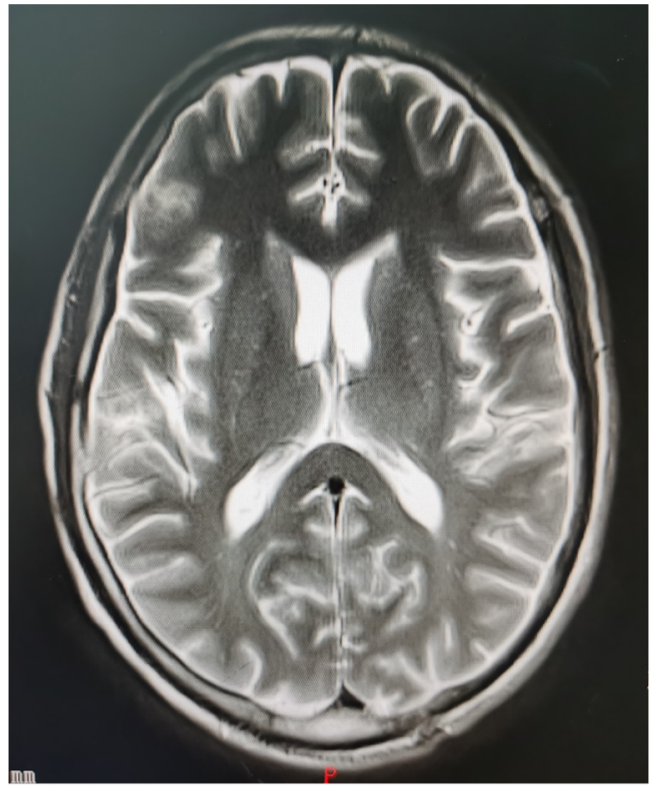

Aug. 2018

pre-osimertinib

Figure S1 Magnetic resonance imaging (MRI) results were normal in August 2018. 